\title{
Smart Oil Fields and Mining Complexes
}

\author{
Louis J. Durlofsky ${ }^{1}$ - Roussos Dimitrakopoulos ${ }^{2}$
}

Published online: 5 April 2017

(C) International Association for Mathematical Geosciences 2017

The optimal development, production and long-term management of petroleum reservoirs and mining complexes are essential for the efficient and sustained use of society's critical resources. Advanced stochastic simulation methods and optimization techniques are used in research, and increasingly in practice, for oil field development and production forecasting, as well as for strategic mine planning and production scheduling. Despite differences in the specific engineering aspects between oil fields and mines or mining complexes, there are broad technical and computational frameworks that are suitable for addressing problems in both fields. Important examples are the use of stochastic simulation methods to quantify uncertainty in the description of petroleum reservoirs and mineral deposits, and the application of robust optimization techniques to determine the best production strategies under geological uncertainty.

In order to highlight and advance interactions in research and development between these two fields, this Special Issue provides technical papers that apply concepts and approaches that we believe will be applicable to both petroleum reservoirs and mining complexes. The Special Issue starts with a paper by Benndorf and Jansen that describes closed-loop approaches as applied within both problem domains. Closedloop treatments include both data assimilation and production optimization, and thus define overall frameworks for optimal operations. In the next paper, Aliyev and Durlofsky define a multilevel (multi-fidelity) optimization procedure for the development of oil reservoirs under geological uncertainty. This is followed by a paper by Goodfellow and Dimitrakopoulos that addresses the simultaneous stochastic optimization of mining complexes and mineral value chains. Next, Liu and Reynolds introduce new

\footnotetext{
Roussos Dimitrakopoulos roussos.dimitrakopoulos@gmail.com

1 Stanford University, Stanford, CA, USA

2 McGill University, Montreal, QC, Canada
} 
treatments for multiobjective optimization, with application to problems involving geological uncertainty. The Special Issue concludes with a paper by Lamghari that surveys the methods used in both application areas and discusses research potential in the fields of optimal mine planning and oil field development.

We hope that readers enjoy this Special Issue, and that research interactions between the two fields expand to their mutual benefit.

Louis J. Durlofsky

and

Roussos Dimitrakopoulos 